Review began 01/17/2022 Review ended 02/01/2022 Published 02/18/2022

๑) Copyright 2022

Gammel et al. This is an open access article distributed under the terms of the Creative Commons Attribution License CCBY 4.0., which permits unrestricted use, distribution, and reproduction in any medium, provided the original author and source are credited.

\section{Controlled Substance Use and Clinical Outcomes of Elderly Patients After a Fall}

\author{
Lauren B. Gammel ${ }^{1}$, Matthew Leonard ${ }^{2}$, Hannah Wheeler ${ }^{2}$, Ha Linh ${ }^{3}$, Bracken Burns Jr. ${ }^{4}$
}

1. General Surgery, East Tennessee State University-Quillen College of Medicine, Johnson City, USA 2. Trauma, Johnson City Medical Center, Johnson City, USA 3. Public Health, East Tennessee State University-Quillen College of Medicine, Johnson City, USA 4. Surgery, East Tennessee State University-Quillen College of Medicine, Johnson City, USA

Corresponding author: Bracken Burns Jr., burnsjb@etsu.edu

\section{Abstract}

Controlled substance use, particularly among the rising elderly population, places these patients at a much higher risk of falls, injuries, and hospitalization. This study examines the association between preinjury controlled substance prescription and clinical outcomes of older adults after a ground-level fall. A total of 5,930 patients were included. Their home medication list was analyzed to record active opioids, benzodiazepines, narcotics, or other substances defined as controlled according to the Drug Enforcement Agency. Almost half (45\%) of the patients were taking controlled substances. Sixty-seven percent of those were females. Total hospital days, Injury Severity Score (ISS), and mortality outcomes were not significantly different between groups. However, intensive care unit (ICU) days, days on mechanical ventilation (MV), and discharge destination were significantly different for patients taking controlled substances versus those not taking controlled substances. Patients taking controlled substances were more likely to be discharged to short- and long-term care facilities versus patients not taking controlled substances $(\mathrm{P} \leqslant 0.001)$.

Categories: Pain Management, Trauma, Substance Use and Addiction

Keywords: fall, outcomes, narcotics, controlled substance, geriatric, trauma

\section{Introduction}

The United States is currently experiencing an opioid crisis. In 2016, there were more than 42,000 opioidrelated overdose deaths in the United States, 115 deaths per day. Nearly, 7,000 of these deaths occurred in five states in the Appalachian region, Alabama, Kentucky, Ohio, Tennessee, and West Virginia [1]. Our center is located in Northeast Tennessee and the Southern Appalachia region. Polypharmacy and controlled substance use among the elderly patient population is of increasing concern. It has been reported that there are 94.4 opioid prescriptions per 100 persons in this population [2].

The prevalence of controlled substance use among trauma patients is thought to be high. A 2013 study in a Kentucky trauma center reported that $20 \%$ of their trauma patient population was taking one or more controlled substance before their injury [3]. Previous work from our institution showed a $29.9 \%$ positive rate of controlled substance use among trauma patients [4]. Falls are the leading cause of fatal injury and the most common cause of nonfatal trauma-related hospital admissions among older adults nationally [5]. The leading cause of trauma at our institution is a ground-level fall, which is more common among the elderly. We have observed a significant amount of preinjury controlled substance prescriptions among our elderly trauma patient population.

Numerous studies have attempted to define the impact of opioid use, specifically on patient outcomes. Multiple studies have shown controlled substance use to be associated with higher rates of mechanical ventilation (MV) and more time in the intensive care unit (ICU) $[3,6,7]$. Two recent reports specifically studied these outcomes in the trauma patient population with no consideration given to patient age [6,7]. Other studies showing this type of outcome correlation have been done in medical and surgical ICU patients without looking specifically at age or previous trauma. In geriatric patients, falls account for the most common cause of traumatic brain injury, death, and injury overall [8]. In 2015 alone, the total national medical costs for falls totaled more than $\$ 50$ billion [9]. Another study in 2015 concluded that preinjury opioid users in the general trauma population are more likely to have presented with a fall as their mechanism of injury [10]. The rate of prescribed controlled substances (especially among geriatric patients, who are more likely to suffer a fall) is of persistent concern both regionally in Appalachia and nationally.

The Geriatric Trauma Committee describes frailty, age-specific alterations in physiologic response to shock, delirium, anticoagulants, and other medications as major factors leading to poor outcomes in geriatric trauma patients [8]. The elderly trauma patient population continues to grow rapidly. This is a vulnerable patient population with unique problems complicating the spectrum of their post-trauma care. Due to the significant burden of this injury mechanism on our elderly patients and our healthcare system overall, we directed our study toward this increasingly important subset of trauma patients. 


\section{Cureus}

The objectives of the study were to examine the association between controlled substance use, risk, and clinical outcomes with mortality and discharge disposition of fall-related injury in older adults. We hypothesized that patients who fell with prescriptions filled for controlled substances were more likely to experience longer length of hospital stay, ICU stay, and days requiring MV.

\section{Materials And Methods}

This is a single-center retrospective review using data from the trauma registry at the Johnson City Medical Center in Johnson City, Tennessee. We included all patients with ground-level falls (GLFs) aged 60 years and older during a seven-year period between January 1, 2011, and December 31, 2017. The Institutional Review Board (IRB) approved waiver of consent. Patient home medication lists were analyzed by pharmacy reconciliation. Active opioids, benzodiazepines, narcotics, or other substances defined within schedules one through five according to the Drug Enforcement Agency controlled substance schedules 2019 revision were the focus of this study.

Statistical significance was set at $\mathrm{P}<0.05$. Chi square analysis was used to test significance for discharge destination to short- and long-term care facilities, mortality, and gender. $\mathrm{T}$ tests were performed to identify any significance between age, total hospital days, intensive care unit days, mechanical ventilation, and injury severity score. Logistic regression was used to determine if the number of controlled substance on home medication lists showed any significant increase in mortality. Statistical tests were completed using Excel v2108 (Microsoft, USA) and JASP v0.14.1.0 software.

\section{Results}

There were a total of 5,930 patients in this study. A total of 3,259 (55\%) were not taking a controlled substance, and 2,671 (45\%) were taking one or more controlled substances. Of the 2,671 patients taking controlled substances, 1,646 (61\%) patients were on one controlled substance, 835 (31\%) patients were on two controlled substances, and 190 (8\%) patients were on three or more controlled substances. Sixty-seven percent of females were taking controlled substances, while males were numbered at $33 \%$. Females were more common in every group. Combination hydrocodone-acetaminophen was the most common controlled substance (686), followed by alprazolam (576) and lorazepam (535). A total of 56 controlled substances or combination of controlled substances were observed on patients' home medication list. A comprehensive table of all controlled substances found on patients' home medication lists is shown in the Appendix, Table 3. The top 10 controlled substances found on patients' home medication list can be seen in Table 1 .

\begin{tabular}{|l|l|}
\hline Name of Substance & Number Found on Patients Home Medication Lists \\
\hline Hydrocodone with acetaminophen & 686 \\
\hline Alprazolam & 576 \\
Lorazepam & 535 \\
\hline Tramadol & 371 \\
Hydrocodone & 297 \\
Clonazepam & 229 \\
Zolpidem & 203 \\
Oxycodone with acetaminophen & 176 \\
Oxycodone & 151 \\
Diazepam & 112 \\
\hline
\end{tabular}

TABLE 1: Top 10 controlled substances found on patients' home medication list.

There was no difference in age between the two study groups. In fact, the mean age was identical for both groups. Injury Severity Score (ISS), gender, and hospital length of stay (LOS) also showed no difference and were nearly identical. Patients were more likely to experience longer ICU days (4) taking controlled substances versus taking no substances (3) $(\mathrm{P}=0.01)$. Patients were also more likely to experience longer mechanical ventilator days (MVD) (7) taking controlled substances versus taking no substances (4), $(\mathrm{P}=0.02)$ (Table 2). Patients taking controlled substances were more likely to be discharged to short- and long-term care facilities versus patients not taking controlled substances $(\mathrm{P}=0.001)$ (Table 2). 


\section{Cureus}

\begin{tabular}{|c|c|c|c|}
\hline & No Controlled Substances & Controlled Substances & $P$ Value \\
\hline \multicolumn{4}{|l|}{ Age } \\
\hline $\mathrm{N}$ & 3,259 & 2,368 & 0.279 \\
\hline Mean & 76 & 76 & \\
\hline Standard deviation & 8.323 & 8.215 & \\
\hline \multicolumn{4}{|l|}{ Injury Severity Score } \\
\hline $\mathrm{N}$ & 3,259 & 2,665 & 0.928 \\
\hline Mean & 9 & 9 & \\
\hline Standard deviation & 5.816 & 5.874 & \\
\hline \multicolumn{4}{|l|}{ Gender } \\
\hline Male N (\%) & $1,069(55 \%)$ & $876(45 \%)$ & 0.997 \\
\hline Female & $3,259(55 \%)$ & $2,671(45 \%)$ & \\
\hline \multicolumn{4}{|l|}{ Total hospital days } \\
\hline $\mathrm{N}$ & 3,259 & 2,671 & 0.314 \\
\hline Mean & 5 & 5 & \\
\hline Median/IQR hospital days & $4 \mathrm{IQR}=3$ & $4 \mathrm{IQR}=3$ & \\
\hline Standard deviation & 5.000 & 5.018 & \\
\hline \multicolumn{4}{|l|}{ Total ICU days } \\
\hline $\mathrm{N}$ & 406 & 353 & .012 \\
\hline Mean & 3 & 4 & \\
\hline Standard deviation & 3.070 & 5.416 & \\
\hline \multicolumn{4}{|l|}{ Total vent days } \\
\hline $\mathrm{N}$ & 87 & 90 & 0.028 \\
\hline Mean & 4 & 7 & \\
\hline Standard deviation & 5.125 & 8.716 & \\
\hline \multicolumn{4}{|l|}{ Discharge disposition } \\
\hline Short- and long-term care facilities & $(66 \%)$ & $(70 \%)$ & $<0.001$ \\
\hline No extended facility & $(34 \%)$ & $(30 \%)$ & \\
\hline
\end{tabular}

TABLE 2: Descriptive statistics for demographics and discharge disposition.

IQR: interquartile range.

Regression analysis showed that patients taking controlled substances had a weak positive correlation between ISS and MVD ( $\mathrm{P}=0.04)$ and there was a moderate correlation between ICU, length of stay (LOS), and MVD ( $\mathrm{P} \leqslant 0.001)$. Multiple logistic regression revealed that there was no significance in mortality between patients taking controlled substances versus those not taking them. However, further analysis showed that increased ISS $(\mathrm{P} \leqslant 0.001)$, increased ICU, LOS $(\mathrm{P} \leqslant 0.001)$, and increased age $(\mathrm{P} \leqslant 0.001)$ were all statistically significantly associated with increased mortality.

\section{Discussion}

The use of controlled substances among trauma patients at our rural Level 1 Trauma Center in Northeast Tennessee had previously been reported as high as $29.9 \%$, regardless of age [4]. We now report an alarmingly high prevalence of controlled substance prescription among the elderly GLF patients in our region (45\%). 


\section{Cureus}

Previously, the published literature has shown negative clinical outcomes associated with preinjury opioid or controlled substance use in the trauma population including increased hospital length of stay, increased ICU length of stay, increased need for MV, and in-hospital mortality [3,7,10,11]. Only one of these studies has been aimed directly toward the elderly trauma patient population [8]. Additionally, there are very limited data comparing outcomes based on a similar injury mechanism such as a ground-level fall.

Our results show that the geriatric GLF patient's use of controlled substances is associated with increased average length of ICU stay, increased average days requiring MV, and an increased likelihood of requiring posthospital short- and long-term facility placement upon discharge. In contrast to some current publicized literature, we found no significance between mortality or ISS and preinjury controlled substance use. Based on these findings, it does not appear that preinjury controlled substance prescription places geriatric patients at any higher risk for increased ISS or mortality but did portend worse outcomes following equivalent injury mechanisms. Additionally, other studies have shown that the use of multiple controlled substances by the same patient resulted in longer hospital and longer ICU stays, though we did not have similar findings [7]. When comparing patients taking one versus two or three controlled substances, there was no statistically significant difference in these parameters in this study.

We recognize that there are limitations to this study. Due to the retrospective nature of this analysis, we were unable to evaluate or analyze current or active use of controlled substances. Additionally, we were unable to ascertain the indication of these prescription medications. This information could have provided an indicator of high-risk status, rather than a causal relationship of these prescription drugs.

Nonprescription drug intake was also unfortunately unable to be reviewed in the retrospective process, though future investigations with this purpose in mind would be intriguing. Additionally, we did not observe the timeframe from when a patient was prescribed their medication/s until the time of their accident. We feel that further evaluation of patients' outcomes with regard to specific injury characteristics or the anatomic area of injury (i.e., the chest or intracranial) could prove to be an interesting and beneficial effort in the future.

At our institution, this information will be shared with the injury prevention coordinator who works with seniors regularly on fall prevention. These results have important clinical implications for the trauma surgeon as well as all physicians prescribing controlled substances as we attempt to improve geriatric healthcare. Our data and the preventative implications of our results will be communicated across educational platforms aimed toward primary care physicians within our hospital system. Early recognition of these higher-risk elderly patients and anticipation of their unique needs with strategic protocols will hopefully improve our clinical outcomes and resource allocation.

Evidence of the correlation between controlled substance use and clinical outcomes of the geriatric trauma patient allows clinicians to identify patients most at risk for adverse outcomes and to plan management accordingly. This knowledge could lead to improved triage protocols, risk stratification, and subsequent management of the injured elderly patient under the physiologic influences of controlled substances. Further investigations should be directed at improving ICU care and disposition planning of geriatric trauma patients taking controlled substances preinjury.

\section{Conclusions}

Ground-level fall patients aged 60 years and older who were prescribed controlled substances preinjury experienced increased ICU stay, mechanical ventilation days, and an increased likelihood of requiring posthospital short- and long-term facility placement upon discharge when compared to those not taking controlled substances. Preinjury controlled substance prescription was not associated with geriatric patients at higher risk for increased ISS or mortality but is associated with longer ICU days, MV days, and discharge to short- and long-term care facilities following similar injury mechanisms.

\section{Appendices}

\section{Appendix}

Name of Controlled Substance

Alprazolam

Atropine

Belladonna

Buprenorphine with naloxone

Carisoprodol

Chlordiazepoxide 


\title{
Cureus
}

\author{
Clonazepam \\ Clorazepate \\ Clorazepate dipotassium \\ Codeine \\ Codeine with acetaminophen \\ Codeine with guaifenesin \\ Codeine with Tylenol \\ Codeine with promethazine \\ Diazepam \\ Dihydrocodeine \\ Diphenoxylate with atropine \\ Dronabinol \\ Eszopiclone \\ Fentanyl \\ Fentanyl patch \\ Fiorinal \\ Flurazepam \\ Hydrocodone \\ Hydrocodone bitartrate \\ Hydrocodone with acetaminophen \\ Hydrocodone with chlorpheniramine \\ Hydrocodone with ibuprofen \\ Hydromorphone \\ Lacosamide \\ Lisdexamfetamine \\ Lorazepam \\ Meperidine \\ Meprobamate \\ Methadone \\ Methylphenidate \\ Modafinil \\ Morphine \\ Oxazepam \\ Oxycodone \\ Oxycodone with acetaminophen \\ Oxymorphone \\ Pentazocine with naloxone \\ Perampanel \\ Phenobarbital \\ Phentermine
}




\section{Cureus}

Pregabalin
Propoxyphene with acetaminophen
Temazepam
Theophylline
Topiramate
Tramadol
Tramadol with acetaminophen
Triazodam
Zaleplon

TABLE 3: Controlled substances found on patients' home medication list.

\section{Additional Information Disclosures}

Human subjects: Consent was obtained or waived by all participants in this study. East Tennessee State University Institutional Review Board issued approval 0919.2sw. This study received waiver of the Health Insurance Portability and Accountability Act (HIPAA) and consent and was approved by the East Tennessee State University and Quillen VA Medical Center (ETSU/VA) Medical IRB. . Animal subjects: All authors have confirmed that this study did not involve animal subjects or tissue. Conflicts of interest: In compliance with the ICMJE uniform disclosure form, all authors declare the following: Payment/services info: All authors have declared that no financial support was received from any organization for the submitted work. Financial relationships: All authors have declared that they have no financial relationships at present or within the previous three years with any organizations that might have an interest in the submitted work. Other relationships: All authors have declared that there are no other relationships or activities that could appear to have influenced the submitted work.

\section{References}

1. U.S. Department of Health and Human Services: Opioid use in Medicare Part D in states in the Appalachian region. (2019). Accessed: May 15, 2020: https://oig.hhs.gov/reports-andpublications/workplan/summary/wp-summary-0000354.asp.

2. Centers for Disease Control and Prevention: U.S. state opioid dispensing rates, 2017 . (2017). Accessed: September 30, 2021: https://www.cdc.gov/drugoverdose/rxrate-maps/state2017.html.

3. Cannon R, Bozeman M, Miller KR, Smith JW, Harbrecht B, Franklin G, Benns M: The prevalence and impact of prescription controlled substance use among injured patients at a Level I trauma center. J Trauma Acute Care Surg. 2014, 76:172-5. 10.1097/TA.0b013e3182ab10de

4. Proctor R, Taylor MP, Quinn M, Burns B Jr: Alarming rate of substance use in motor vehicle collisions at an Appalachian trauma center. Cureus. 2020, 12:e11863. 10.7759/cureus.11863

5. National Council on Aging: Get the facts on falls prevention . (2021). Accessed: December 1, 2021: https://www.ncoa.org/article/get-the-facts-on-falls-prevention.

6. University of Utah Health: Benzodiazepine sedatives linked to higher rates of mortality compared to propofol. (2014). Accessed: May 23, 2020: https:/healthcare.utah.edu/publicaffairs/news/2014/09/09-30-14Benzodiazepines-Sedatives-Mortality.php.

7. Cheng V, Inaba K, Johnson M, et al.: The impact of pre-injury controlled substance use on clinical outcomes after trauma. J Trauma Acute Care Surg. 2016, 81:913-20. 10.1097/TA.0000000000001229

8. Kozar RA, Arbabi S, Stein DM, et al.: Injury in the aged: geriatric trauma care at the crossroads . J Trauma Acute Care Surg. 2015, 78:1197-209. 10.1097/TA.0000000000000656

9. Florence CS, Bergen G, Atherly A, Burns E, Stevens J, Drake C: Medical costs of fatal and nonfatal falls in older adults. J Am Geriatr Soc. 2018, 66:693-8. 10.1111/ggs.15304

10. Pandya U, O'Mara MS, Wilson W, Opalek J, Lieber M: Impact of preexisting opioid use on injury mechanism, type, and outcome. J Surg Res. 2015, 198:7-12. 10.1016/j.jss.2015.05.033

11. Daoust R, Paquet J, Moore L, et al.: Recent opioid use and fall-related injury among older patients with trauma. CMAJ. 2018, 190:E500-6. 10.1503/cmaj.171286 